\title{
The Existence of Thick Triangulations - An "Elementary" Proof
}

\author{
Emil Saucan* and Meir Katchalski
}

Mathematics Department, Technion, Technion City, Haifa 32000, Israel

\begin{abstract}
We provide an alternative, simpler proof of the existence of thick triangulations for noncompact $C^{1}$ manifolds. Moreover, this proof is simpler than the original one given in [1], since it mainly uses tools of elementary differential topology. The role played by curvatures in this construction is also emphasized.
\end{abstract}

Keywords: Thick triangulation, $C^{1}$ manifolds, cut locus.

\section{INTRODUCTION}

The existence of the so called "thick" or "fat" triangulations is important both in Pure Mathematics, in Differential Geometry (where it plays a crucial role in the computation of curvatures for piecewise-flat approximations of smooth Riemannian manifolds, with applications to Regge Calculus, see [2]); and in Geometric Function Theory (mainly in the construction of quasimeromorphic mappings, see [1, 3-7]). Thick triangulations play also an important role in many applications, mainly in Computational Geometry, Computer Graphics, Image Processing and their related fields (see, [8-13], to name just a few).

Recall that "thick" or "fat" triangulations are defined as follows:

Definition 1.1. Let $\tau \subset \mathbb{R}^{n} ; 0 \leq k \leq n$ be a $k$-dimensional simplex. The thickness $\varphi$ of $\tau$ is defined as being:

$$
\varphi=\varphi(\tau)=\inf _{\substack{\sigma<\tau \\ \operatorname{dim} \sigma=j}} \frac{\operatorname{Vol}_{j}(\sigma)}{\operatorname{diam}^{j} \sigma} .
$$

The infimum is taken over all the faces of $\tau, \sigma<\tau$, and $\operatorname{Vol}_{j}(\sigma)$ and diam $\sigma$ stand for the Euclidian $j$-volume and the diameter of $\sigma$ respectively. (If $\operatorname{dim} \sigma=0$, then $\operatorname{Vol}_{j}(\sigma)=1$, by convention).

A simplex $\tau$ is $\varphi_{0}$-thick, for some $\varphi_{0}>0$, if $\varphi(\tau) \geq \varphi_{0}$. A triangulation (of a submanifold of $\mathbb{R}^{n}$ ) $\mathcal{T}=\left\{\sigma_{i}\right\}_{i \in \mathrm{I}}$ is $\varphi_{0}$-thick if all its simplices are $\varphi_{0}$-thick. A triangulation $\mathcal{T}=\left\{\sigma_{i}\right\}_{i \in \mathrm{I}}$ is thick if there exists $\varphi_{0} \geq 0$ such that all its simplices are $\varphi_{0^{-}}$ thick.

The definition above is the one introduced in [14]. For some different, yet equivalent definitions of thickness, see [1, 4, 15-17].

For $C^{\infty}$ Riemannian manifolds without boundary the existence has been proved by Peltonen [1]. This result was

*Address correspondence to this author at the Mathematics Department, Technion, Technion City, Haifa 32000, Israel;

E-mail: semil@tx.technion.ac.il

1991 Mathematics Subject Classification. AMS Classification. 53A07, 57R05, 57M60, A05. extended by the first author to include manifolds of lower differentiability class with boundary [18] and to a large class of orbifolds [7].

Peltonen's proof is based on the construction of an exhaustion of the manifold using a delicate curvature-based argument, both to decide the "size" of the compact "pieces" (i.e. of the elements of the exhaustion) and to chose the mash of the triangulation (see [1] and also Section 2). The technique used in [18] is much more elementary, using only the Differential Topology apparatus (and results) of [17]. This discrepancy between methods creates a kind of "esthetic asymmetry" that naturally gives rise to the following question:

Question 1.2. Is it possible to prove the existence of thick triangulations for non-compact manifolds using only techniques of Elementary Differential Topology?

We shall prove that the answer to the question above is positive by showing that the "mashing" technique of thick triangulations developed in [18] allows us to discard the curvature considerations of the original proof.

However, in discarding the curvature-related information, one also loses geometric intuition and, with it, any possibility of applying the technique in any non-trivial, concrete case. Hence, the next question ensues immediately:

Question 1.3. Can one recover the Differential Geometric information (i.e. curvatures) from the constructed PL triangulation?

We show that, again, the answer is affirmative, and it follows from the results of [14]. Moreover, we indicate how this approach can also be simplified using tools that may be considered more "elementary".

The remainder of the paper is organized as follows: In the next Section we briefly sketch, for the benefit of the reader and for the sake of the paper's self-containment, the main steps in the proofs of the main results in [1] and [18]. In Section 3, we show how our result in [18] allows us to give a simpler, "elementary" proof of Peltonen's theorem. Finally, in the last section, we discuss the role played by curvature in our simplified proof and indicate how, using our method, one still can recapture the Differential Geometric information encoded in Peltonen's proof. 


\section{BACKGROUND}

We bring below a very brief sketch of the methods used in proving the existence of thick triangulations. We concentrate mainly on those aspects that are pertinent to our present study.

\subsection{Open Riemannian Manifolds}

First, a number of necessary definitions:

Let $M^{n}$ denote an $n$-dimensional complete Riemannian manifold, and let $M^{n}$ be isometrically embedded into $\mathbb{R}^{v}$ (" $v$ "s existence is guaranteed by Nash's Theorem - see, e.g. [1]).

Let $\mathbb{B}^{v}(x, r)=\left\{y \in \mathbb{R}^{v \mid} d_{\text {eucl }}<r\right\} ; \partial \mathbb{B}^{v}(x, r)=\mathbb{S}^{v-1}(x, r)$. If $x \in M^{n}$, let $\sigma^{n}(x, r)=M^{n} \cap \mathbb{B}^{v}(x, r), \beta^{n}(x, r)=\exp _{x}\left(\mathbb{B}^{n}(0, r)\right)$, where: $\exp _{x}$ denotes the exponential $n$ map: $\exp _{x}: T_{x}\left(M^{n}\right) \rightarrow$ $M^{n}$, and where $\mathbb{B}^{n}(0, r) \subset T_{x}\left(M^{n}\right)$, B $(0, r)=\left\{y \in \mathbb{R}^{n \mid} d_{\text {eucl }}(y\right.$, $0)<r\}$.

The following definitions generalize in a straightforward manner classical ones used for surfaces in $\mathbb{R}^{3}$ :

\section{Definition 2.1.}

$\mathbb{S}^{v-1}(x, r)$ is tangent to $M^{n}$ at $x \in M^{n}$ iff there exists $\mathbb{S}^{n}(x, r) \subset \mathbb{S}^{v-1}(x, r)$, such that $T_{x}\left(\mathbb{S}^{n}(x, r)\right) \equiv T_{x}\left(M^{n}\right)$.

Let $l \subset \mathbb{R}^{v}$ be a line, then $l$ is secant to $X \subset M^{n}$ iff $\mid l \cap$ $X \mid \geq 2$.

\section{Definition 2.2.}

(1) $\mathrm{S}^{\nu-1}(x, \rho)$ is an osculatory sphere at $x \in M^{n}$ iff:

(a) $\mathbb{S}^{v-1}(x, \rho)$ is tangent at $x$; and

(b) $\mathbb{B}^{n}(x, \rho) \cap M^{n}=\varnothing$.

(2) Let $X \subset M^{n}$. The number $\omega=\omega_{X}=\sup \left\{\rho>0 \mid \mathbb{S}^{\nu-1}(x\right.$, $\rho$ ) osculatory at any $x \in X\}$ is called the maximal osculatory (tubular) radius at $X$.

Remark 2.3. There exists an osculatory sphere at any point of $M^{n}$ (see [19]).

\section{Definition 2.4.}

Let $U \subset M^{n}, U \varnothing$, be a relatively compact set, and let $=T$ $=\cup_{x \in \bar{U}} \sigma\left(x, \omega_{U}\right)$. The number $\kappa_{U}=\max \left\{r \mid \sigma^{n}(x, r)\right.$ is connected for all $r \leq \omega_{U}, x \in \bar{T}$, is called the maximal connectivity radius at $U$.

Note that the maximal connectivity radius and the maximal osculatory radius are interconnected by the following inequality ([1], Lemma 3.1):

$$
\omega_{U} \leq \frac{\sqrt{3}}{3} \kappa_{U} .
$$

We are now able to present the main steps of Peltone's proof, which generalizes both the result and method of proof of Cairns [19]:

(a) Construct an exhaustion of $M^{n}$ by compact sets $\left\{E_{i}\right\}$, generated by the pair $\left(U_{i}, \eta_{i}\right)$, where:

(i) $\quad U_{i}$ is the relatively compact set $E_{i} \mid \bar{E} i_{-1}$ and

(ii) $\eta_{i}$ is a number that controls the fatness of the simplices of the triangulation of $E_{i}$, to be constructed in (2) below, such that it will not differ to much on adjacent simplices, i.e.:

(ii $\left.{ }_{1}\right) \quad$ The sequence $\left(\eta_{i}\right)_{i \geq 1}$ descends to 0 ;

(ii $) \quad 2 \eta_{i} \geq \eta_{i-1}$.

The numbers $\eta_{i}$ are chosen such that they satisfy the following bounds:

$$
\eta_{i} \leq \frac{1}{4} \min \left\{\omega_{\bar{U}_{i-1}}, \omega_{\bar{U}_{i}}, \omega_{\bar{U}_{i+1}}\right\}
$$

(b) (i) Produce a maximal set $A,|A| \leq \kappa_{0}$, such that $A \cap U_{i}$ satisfies:

(i $i_{1}$ a density condition, namely:

$d(a, b) \geq \eta_{i} / 2$, for all $a, b \in A$, and for all $i \geq 1$;

$\left(i_{2}\right) \quad$ a "gluing" condition for $U_{i}, U_{i+1}$, i.e. their intersection is large enough.

Note that according to the density condition $\left(i_{1}\right)$, the following holds: For any $i$ and for any $x \in \bar{U}_{i}$, there exists $a$ $\in A$ such that $d(x, a) \leq \eta_{i} / 2$.

(ii) Prove that the Dirichlet complex $\left\{\bar{\gamma}_{i}\right\}$ defined by the sets $A_{i}$ is a cell complex and every cell has a finite number of faces (so that it can be triangulated in a standard manner).

(2) Consider first the dual complex $\Gamma$, and prove that it is a Euclidean simplicial complex with a "good" density. Project then $\Gamma$ on $M^{n}$ (using the normal map). Finally, prove that the resulting complex $\tilde{\Gamma}$ can be triangulated by fat simplices.

\subsection{Manifolds With Boundary}

The idea of the proof in this case is to build first two fat triangulations: $\mathcal{T}_{1}$ of a product neighbourhood $N$ of $\partial M^{n}$ in $M^{n}$ and $\mathcal{T}_{2}$ of int $M^{n}$ (its existence follows from Peltonen's result), and then to "mash" the two triangulations into a new triangulation $\mathcal{T}$, while retaining their thickness (see [18]). While the mashing procedure of the two triangulations is basically the classical one of [17], the triangulation of $\mathcal{T}_{1}$ has been modified, in order to ensure the thickness of the simplices of $\mathcal{T}_{1}$ (see [18], Theorem 2.9). To thicken triangulations one can use either the method used in [14], or, alternatively, the one developed in [5]. For the technical details, see [18].

\section{MAIN RESULT}

The idea of the proof is to use the basic fact that $M^{n}$ is $\sigma$ compact, i.e. it admits an exhaustion by compact submanifolds $\left\{K_{j}\right\}_{j}$ (see, e.g. [21]). This is a standard fact for metrizable manifolds. However, it is conceivable that the "cutting surfaces" $N_{i j}, \cup_{i=1, \ldots . k_{j}} N_{i j}=\partial K_{j}$, are merely $C^{0}$, so even the existence of a triangulation for these hypersurfaces is not always assured, hence a fortiori that of smooth triangulartions. (See. e.g. [20] for a brief review of the results regarding the existence of triangulations).

To show that one can obtain (by "cutting along") smooth hypersurfaces, we briefly review the main idea of the proof 
of the $\sigma$-compactness of $\mathrm{M}^{n}$ (for the full details, see, for example [21]): Starting from an arbitrary base point $\mathrm{x}_{0} \in M^{n}$, one considers the interval $I=I\left(x_{0}\right)=\left\{r>0 \mid \beta^{n}\left(x_{0}, r\right)\right.$ is compact\}, where $\beta^{n}\left(x_{0}, r\right)$ is as in Section 2. If $I=\mathbb{R}, \cup_{1}^{\infty}$ $\beta^{n}(x, n)$, thence $\sigma$-compact. If $I \neq \mathbb{R}$, one constructs the compacts sets $K_{j}, K_{0}=\left\{x_{0}\right\}, K_{j+1}=\cup_{y_{\in} k_{j}} \beta^{n}(y, r(y))$, where $r(y)=1 / 2 \sup I(y)$. Then it can be shown that $M^{n}=\cup_{j \geq 0} K_{j}$, i.e. $M^{n}$ is $\sigma$-compact. The smoothness of the surfaces $N_{i j}$ now follows from Wolter's result [22] regarding the 2-differentiability of the cut locus of the exponential map.

By [15] (see also [16]) the sets $K_{j}$ and $N_{i j}$ admit thick triangulations and, moreover, these triangulations have thickness $\varphi_{1}=\varphi_{1}(n)$ and $\varphi_{2}=\varphi_{2}(n-1)$, respectively. One can thus apply repeatedly the "mashing" technique developed in [18], for collars of $N_{i j}$ in $K_{j}$ and $K_{j+1}, j \geq 0$, rendering a triangulation of $M^{n}$, of uniform thickness $\varphi=\varphi$ (n) $($ see $[14,18])$.

Remark 3.1. Instead of the less known and more complicated method of [15], we could have employed the simpler and more intuitive one in [16]. However, the later one still makes use of the principal curvatures (at each point of) the manifold, thus using this method the "Differential Geometric content", so to speak, of our proof would still be rather higher. Since we strive to obtain a proof that is as elementary as possible, i.e. using only (or mainly) the tools of elementary differential topology, we prefer to adopt the methods of Cairns' earlier work.

Remark 3.2. In some special cases a "purely" Euclidean construction can be achieved, without resorting to embeddings and piecewise-flat (or just piecewise-linear) approximations. Such a construction is provided in [23], where a method of dividing a non-compact hyperbolic manifold into (canonical) Euclidean pieces is described. Note that, moreover, these pieces can be easily subdivided into thick simplices using a number of the methods mentioned above (and in the bibliography).

Remark 3.3. The opposite problem, that of extending a thick triangulation from the interior of a manifold to its boundary, is also worth considering, in itself and for its importance in Geometric Function Theory (see [7]).

\section{CURVATURES}

We conclude with a few brief remarks regarding the role of curvature:, its possible "recovery" in the $P L$ context, and further directions of study.

First, let us note that, manifestly enough, our simplified proof is not "Differential Geometry free", since we used both the exponential map and Wolter's result. Regarding an "elementary" proof, this is evidently a weakness, as is the appeal we have made to Nash's Embedding Theorem (with its complicated Differential Geometric apparatus). One would be tempted to substitute for the balls $\beta^{n}(x, j)$, Euclidean balls $\mathbb{B}^{v}(x, j)$, and replace, if necessary, the surfaces $L_{j k}=\partial \mathbb{B}^{v}(x, j) \cap M^{n}$ by $C^{1}$ surfaces $L_{j}^{*}$ that are $\varepsilon$ close to $L_{j}$. Such an approach would allow us to consider only $C^{1}$ em-beddings, thus permitting us to dispense with the use of Nash's Theorem. Unfortunately, in general, one cannot discard the use of the exponential map (and, consequently, neither can one ignore Wolter's work). Indeed, for wildly embedded manifolds, the simple method above is not applicable. However, if only tame embeddings are considered, than the method above can be employed.

Secondly, let us note that Wolter's method is - obviously - not independent of curvature. Therefore, the curvature considerations do play a decisive role, even if in a more "soft" manner. Moreover, even if applying the very simple and direct method described in the previous paragraph, the curvature plays an intrinsic role in determining the meshes of the triangulations of two adjacent "pieces" $K_{j}$ and $K_{j+1},\left(K_{j}=\right.$ $\left.\mathbb{B}^{v}(x, j) \cap M^{n}\right)$, and their common boundary $L_{j k}^{*}$. For this reason, a "naive" exhaustion of the manifold using Euclidean balls, hence without control of curvature, may prove, in general, to be counterproductive, rendering not a monotone (and even highly oscillating) series of meshes for the members of the exhaustion.

Finally, as noted in the Introduction, the (LipschitzKilling) curvatures have "good" convergence properties, under piecewise-flat (secant) approximations of $M^{n}$. This result of Cheeger et al. (see [14]) is the goal for which they developed the "mashing of triangulations" technique mentioned above. However, a simpler and more direct approach to curvatures computation in $P L$ approximation, along the lines indicated in [24] and based on metric curvatures, is currently in preparation [25].

\section{ACKNOWLEDGMENTS}

The first author wishes to express his gratitude to Professor Shahar Mendelson - his warm support is gratefully acknowledged. He would also like to thank Professor KlausDieter Semmler for bringing to his attention Wolter's work. Second author's research partially supported by the Japan Technion Society Research Fund.

\section{REFERENCES}

[1] Peltonen K. On the existence of quasiregular mappings. Ann Acad Sci Fenn Ser I Math Dissertationes 1992; 85: 48.

[2] Regge T. General relativity without coordinates. Nuovo Cimento 1961; 19: 558-71.

[3] Martio M, Srebro U. On the existence of automorphic quasimeromorphic mappings in $R^{n}$. Ann Acad Sci Fenn Ser A I Math 1977; 3 : 123-30.

[4] Tukia P. Automorphic quasimeromorphic mappings for torsionless hyperbolic groups. Ann Acad Sci Fenn 1985; 10: 545-60.

[5] Saucan E. The existence of quasimeromorphic mappings in dimension 3. Conform Geom Dyn 2006; 10: 21-40.

[6] Saucan E. The existence of quasimeromorphic mappings. Ann Acad Sci Fenn Ser A I Math 2006; 31: 131-42.

[7] Saucan E. Remarks on the existence of quasimeromorphic mappings. Contemp Math 2008; 455: 325-31.

[8] Amenta N, Bern M. Surface reconstruction by Voronoi filtering. Discrete Comput Geom 1999; 22: 481-504.

[9] Bern M, Chew LP, Eppstein D, Ruppert J. Dihedral Bounds for Mesh Generation in High Dimensions. In: Clarkson K, Ed. 6th ACM-SIAM Symposium on Discrete Algorithms Conference Proceedings; San Francisco: ACM-SIAM; 1995; pp. 189-96.

[10] Cheng SW, Dey TK, Ramos EA. Manifold Reconstruction from Point Samples. Proceedings of ACM-SIAM Symposium Discrete Algorithms Conference; Canada: ACM-SIAM; 2005; pp. 1018-27.

[11] Edelsbrunner H. Geometry and topology for mesh generation. Cambridge: Cambridge University Press 2001. 
[12] Pach J, Agarwal PK. Combinatorial Geometry. New York: John Wiley 1995.

[13] Saucan E, Appleboim E, Zeevi YY. Sampling and reconstruction of surfaces and higher dimensional manifolds. J Math Imag Vis 2008; 30(1): 105-23.

[14] Cheeger J, Müller W, Schrader R. On the curvature of piecewise flat spaces. Comm Math Phys 1984; 92: 405-54.

[15] Cairns SS. On the triangulation of regular loci. Ann Math 1934; 35 : 579-87.

[16] Cairns, SS. Polyhedral approximation to regular loci. Ann Math 1936; 37: 409-19.

[17] Munkres, JR. Elementary differential topology. Revised ed. Princeton: Princeton University Press 1966.

[18] Saucan E. Note on a theorem of Munkres. Mediterr J Math 2005; 2(2): 215-29.
[19] Cairns, SS. A simple triangulation method for smooth manifolds. Bull Am Math Soc 1961; 67: 380-90.

[20] Spivak M. A comprehensive introduction to differential geometry I. Boston: Publish or Perish Press 1970.

[21] Thurston W. Three-Dimensional Geometry and Topology. Levy S, Ed. Princeton: Princeton University Press 1997.

[22] Wolter FE. Cut loci in bordered and unbordered riemannian manifolds. PhD. Diss., Dept. of Mathematics, Technical University Berlin 1985.

[23] Epstein DBA, Penner RC. Euclidean decompositions of noncompact hyperbolic manifolds. J Diff Geom 1988; 27: 67-80.

[24] Saucan E. Curvature - Smooth, Piecewise-Linear and Metric. In: Sica G, Ed. What is Geometry? Advanced Studies in Mathematics and Logic Milano: Polimetrica 2006; pp. 237-68.

[25] Saucan E. Triangulations, Quasiregular mappings and $P L-$ differential geometry. Forthcoming.

(C) Saucan and Katchalski; Licensee Bentham Open.

This is an open access article licensed under the terms of the Creative Commons Attribution Non-Commercial License (http://creativecommons.org/licenses/by$\mathrm{nc} / 3.0 /$ ), which permits unrestricted, non-commercial use, distribution and reproduction in any medium, provided the work is properly cited. 